\title{
La fuerza del vacío. El efecto Casimir
}

\author{
R. Esquivel SiRVent*
}

Resumen: En este artículo presentamos algunos aspectos básicos sobre la fuerza de Casimir que resulta de las fluctuaciones del vacío cuántico. Se discute sobre los experimentos recientes para medir esta fuerza, su conexión con las fuerzas de Van der Waals y, finalmente, acerca de las maneras de modular la fuerza de Casimir y cómo inducir torcas de Casimir.

\section{INTRODUCCIÓN}

En 1948, el físico Holandés H. B. Casimir [1] realizó un cálculo simple que demostraba un efecto inesperado. Al colocar dos placas metálicas neutras una enfrente de otra y separadas una distancia $l$, éstas se atraerían con una fuerza que sólo dependía de dos constantes fundamentales la constante de Plank, $\hbar$ y la velocidad de la luz, $c$. También era función del área, $A$, de las placas y de la separación entre éstas de la siguiente manera:

$$
f_{0}=-\frac{\hbar c \pi^{2} A}{240 l^{4}}
$$

Este inocuo resultado inició un campo de estudio vigente hasta nuestros días y que se extiende por varias áreas de la física como teoría de campos, estado sólido, altas energías, cosmología y, más recientemente, nanociencias y nanotecnología.

Para explicar el efecto, cuenta Casimir lo siguiente [2]: "Mencione mis resultados a Niels Bohr, durante una caminata. Le dije estar sorprendido por lo extremadamente simple de la forma del resultado y él murmuró algo sobre la energía de punto cero".

La energía de punto cero es la mínima energía que un sistema puede tener. En el caso del vacío, la teoría cuántica de campos supone que aun a la energía de punto cero, siempre hay ondas electromagnéticas fluctuantes de cualquier frecuencia. Al poner las dos placas paralelas, entre éstas sólo pueden existir ondas que cumplan con las condiciones de frontera. Es decir, ondas que tengan nodos en las placas. Por otro lado, afuera de las placas no hay restricción y pueden existir ondas de cualquier frecuencia. De manera simplista, podemos decir que hay más

FIGURA 1. Hendrik Casimir (1909-2000)

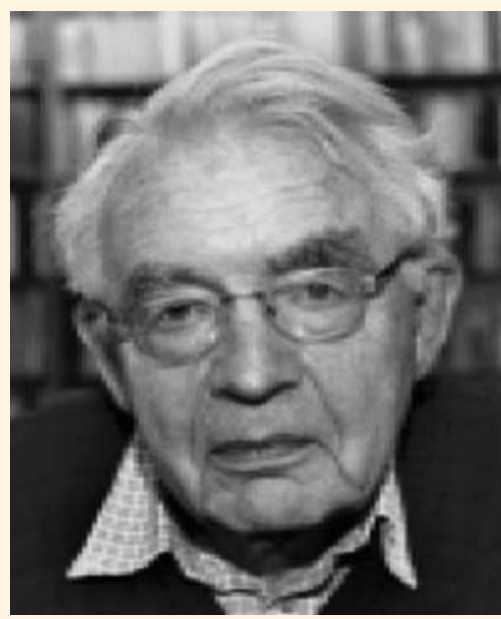

Fuente: Wikipedia.

* Instituto de Física, Universidad Nacional Autónoma de México. 
ondas afuera de las placas que entre ellas o bien que la energía de las ondas electromagnéticas afuera de las placas es mayor que la energía que hay entre las placas. Esta diferencia de energía es la que causa la fuerza de Casimir. En la figura 2, se muestra un diagrama de las ondas electromagnéticas afuera y adentro.

Matemáticamente, el deducir la fuerza de Casimir lleva a problemas de energías infinitas que pueden ser eliminados usando diversas técnicas. A los avezados en matemáticas se les recomienda leer la referencia [3].

FIGURA 2. Diagrama que ilustra el principio del efecto Casimir. Hay más ondas afuera de las placas que entre ellas.

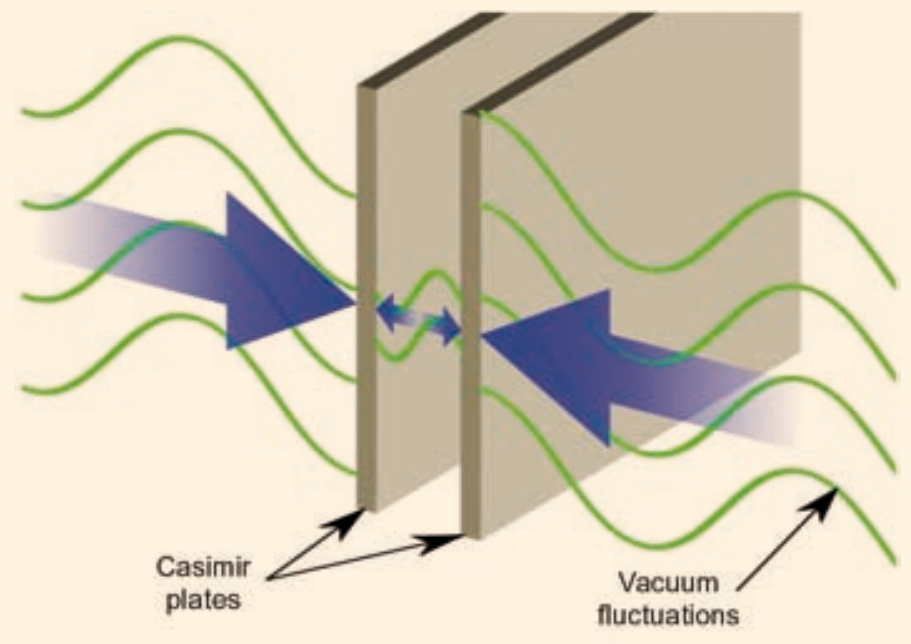

Imagen tomada de Wikipedia.

La formula de Casimir mostrada en la ecuación 1 se dedujo para metales perfectos. Esto es, materiales ideales que reflejan toda la luz, son reflectores perfectos. No existen en la naturaleza, pero nos ayudan a entender la física del fenómeno. ¿Qué pasa si las placas están hechas de un material real? La teoría de Casimir tiene que ser modificada. Esto fue hecho por el físico soviético Evgeny Lifshitz [4], quien desarrolló una teoría bastante complicada pero capaz de predecir la fuerza de Casimir entre materiales arbitrarios. Sin entrar en los detalles del cálculo que se basan en el teorema de fluctuación disipación, nos bastará decir que la fuerza para materiales reales se puede escribir como

$$
f=\eta f_{0}
$$

donde $\eta$ es el factor de reducción que es menor o igual a uno. El valor de uno es para los conductores perfectos.

Por ejemplo, se puede calcular la fuerza entre dos placas de oro, de silicio o de cualquier material. La fuerza de Casimir es mayor para metales. Un ejemplo del cálculo de la fuerza lo presentamos en la figura 3. Para dos placas de oro ( $\mathrm{Au}$ ) se obtiene la 
fuerza más grande [4], para la placa de Au y Si disminuye $\eta$ porque el Si no es un buen conductor y finalmente, al tener las dos placas de Si se obtiene el valor más pequeño para la fuerza. Dicho de otra manera, la teoría de Lifshitz nos permite concluir que entre más luz refleja un material mayor será la fuerza de Casimir.

FIGURA 3. Factor de reducción $\mathrm{f} / \mathrm{fo}$ donde mostramos que la fuerza es mayor para buenos conductores.

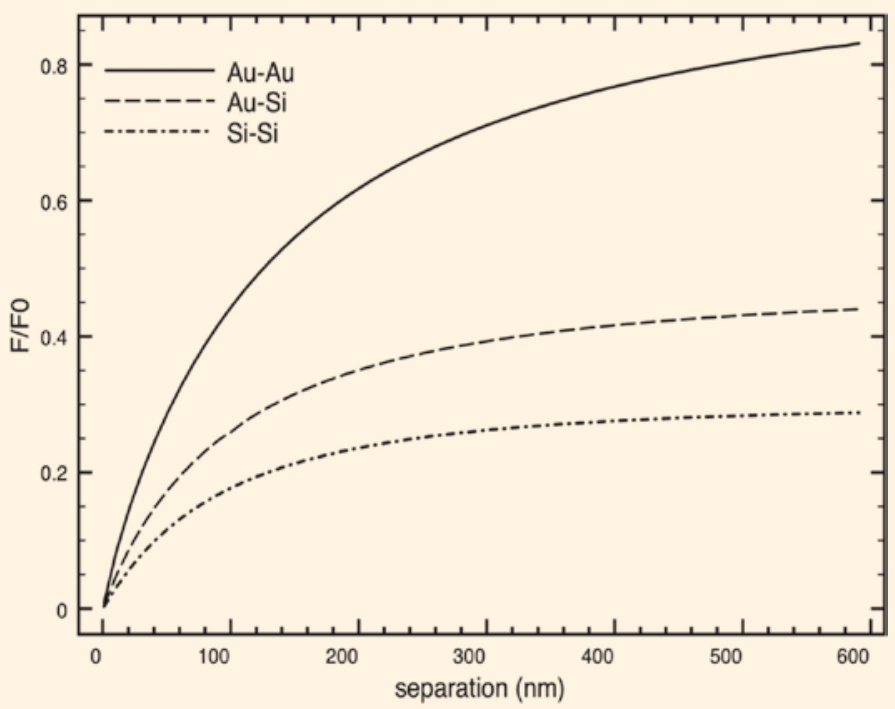

\section{LOS EXPERIMENTOS}

Si bien hubo intentos experimentales para comprobar el efecto Casimir, fue hasta la década de los años 90 del siglo XX que Steve Lamoreaux, utilizando una balanza de torsión [5], logró medir con suficiente precisión la fuerza de Casimir. Casi simultáneamente, Umar Mohideen midió la fuerza utilizando un microscopio de fuerza atómica [6]. El experimento consistió en pegar una esfera de oro a una punta de fuerza atómica y medir la fuerza entre la esfera y una placa de oro. El dispositivo se muestra en la figura 4.

El interés por el efecto Casimir motivó otra serie de experimento y se midió de nuevo la fuerza utilizando micro balanzas de torsión, demostrándose, además, que esta fuerza se podía utilizar en micro y nano máquinas [7]. En la figura 5, se muestra un esquema del funcionamiento de una micro balanza de torsión cuyo tamaño es de aproximadamente 100 millonésimas de metro por lado. Al acercar la esfera, la balanza de torsión se inclina y entre más grande es la fuerza, mayor será la inclinación. De aquí se puede determinar el valor de la fuerza de Casimir.

La fuerza de Casimir se mide en pico Newtons es decir una billonésima de Newton. ${ }^{1}$ Para darnos una idea de esto, la fuerza que ejercemos al oprimir el botón de un bolí-

\footnotetext{
1 Usamos el termino billón en español. Es decir un millón de millones.
} 
Mundo Nano | Artículos | Vol. 4, No. 1, enero-junio, 2011 | www.mundonano.unam.mx

FIGURA 4. Punta de microscopio de fuerza atómica con esfera pegada para la medición de la fuerza de Casimir. Foto tomada de la referencia [6].

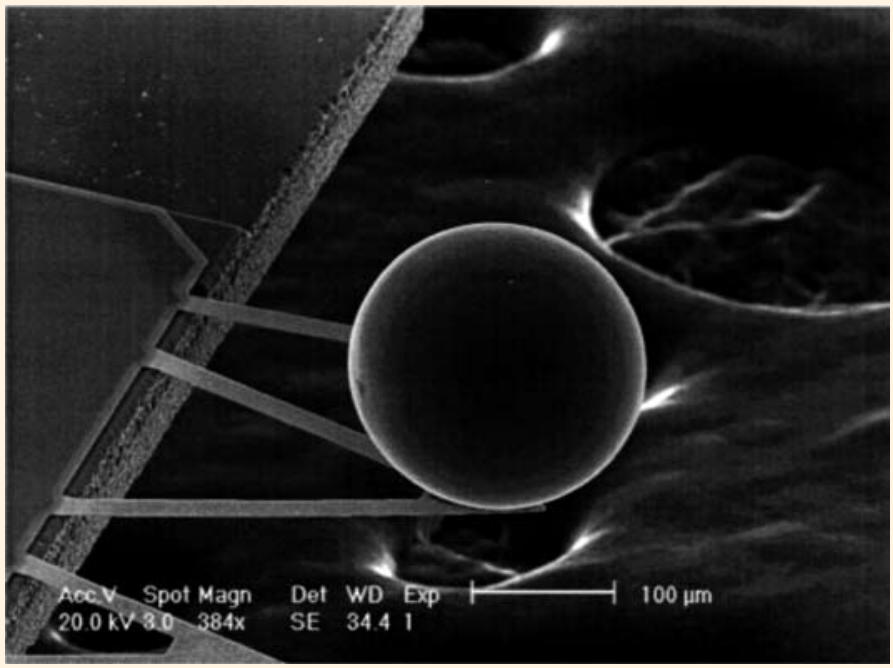

FIGURA 5. Esquema de funcionamiento de una micro balanza de torsión para medir fuerzas de Casimir. Imagen tomada de <www.harvard.deas/capasso $>$.

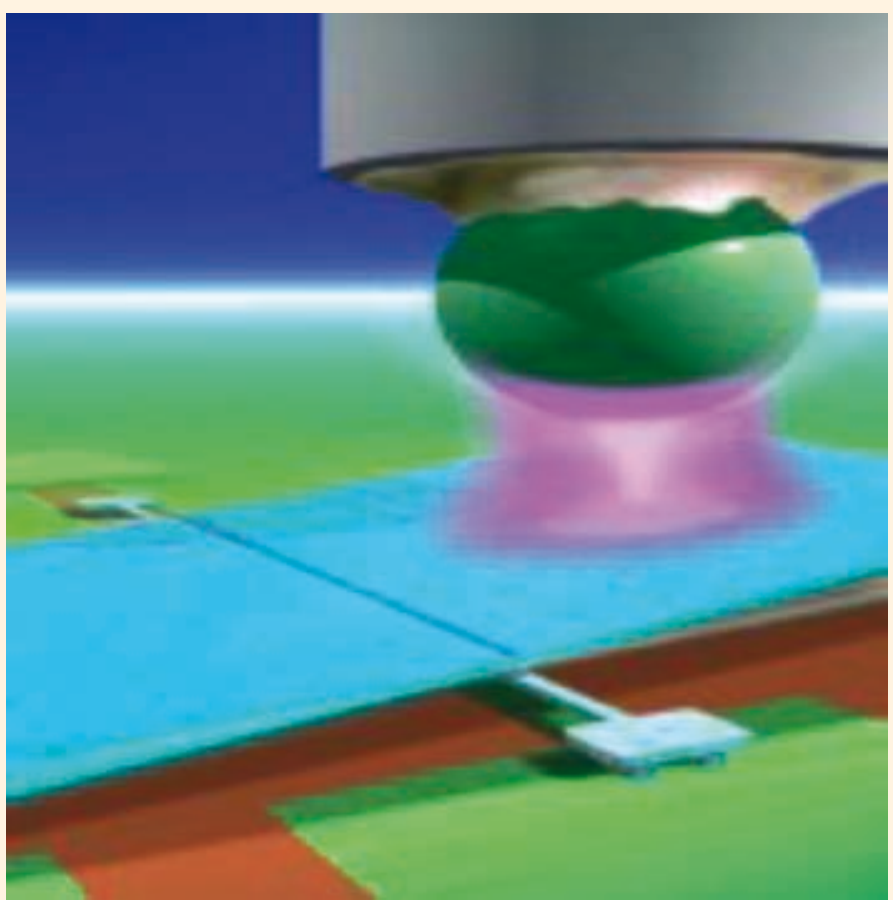


grafo para sacar la punta es de 1 Newton aproximadamente. En la figura 6, se puede observar una curva típica de medición de la fuerza de Casimir. Estas mediciones fueron realizadas con un microscopio de fuerza atómica. En esta figura, los puntos negros son varias mediciones que se realizaron y la línea roja es la predicción usando la fórmula de Lifshitz. Como vemos el promedio de las mediciones coincide con la teoría [8].

FIGURA 6. Ejemplo de mediciones de la fuerza de Casimir utilizando un microscopio de fuerza atómica. Tomado de la referencia [8].

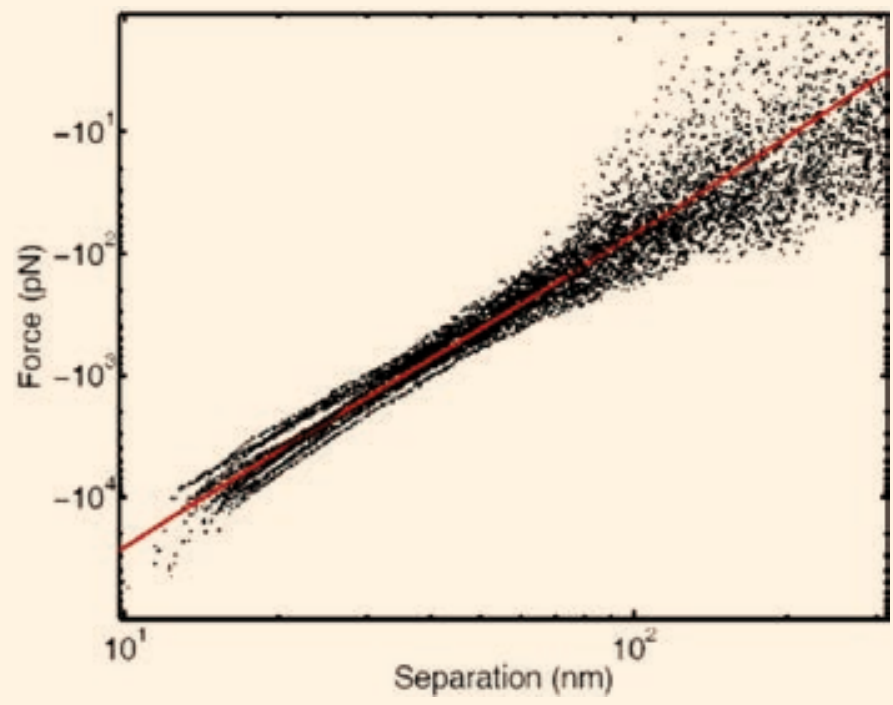

\section{CASIMIR o VAN der WaALS}

Es común en los cursos de química hablar de las fuerzas de Van der Waals. Estas fuerzas fueron propuestas por el físico holandés Johannes Van der Waals, quien propuso que las fuerzas entre moléculas se producían por interacciones tipo dipolar. Por razones históricas y casi absurdas las interacciones dipolares se conocen por varios nombres como fuerzas de London, de Debye, pero todas son de origen dipolar.

Si se conoce la fuerza entre dos moléculas, y las dos placas paralelas de la figura 2 están formadas por moléculas, la pregunta es si se puede encontrar la fuerza entre las moléculas simplemente sumando la fuerza entre las moléculas de una placa con las de la otra placa. Este método propuesto por Hamaker [9], resulta aproximado, pues no toma en cuenta que las moléculas en una placa también interaccionan entre ellas. Es justamente esta limitación la que es solucionada con la fórmula de Lifshitz mencionada anteriormente. Por eso, la teoría de Lifshitz también se conoce como teoría de Van der Waals generalizada. 


\section{LOS SISTEMAS MICRO Y NANO ELECTROMECÁNICOS}

Uno de los avances tecnológicos recientes de los últimos años es la invención de las micro y nano máquinas conocidos como mems y nems. Es decir, dispositivos con partes móviles pero cuyo tamaño es a lo más de unos cientos de micras. Por ejemplo, en la figura 7, vemos una foto de mems que consiste de un engrane acoplado a un cigueñal. El dispositivo de la figura se puede considerar grande, ya que el diámetro del engrane es de unas 50 micras. Actualmente, el lograr dispositivos con partes móviles de unas cuantas micras ya es una realidad.

FIGURA 7. Micro engrane acoplado a cigueñal de una micro máquina. Foto tomada de Sandia Nat. Laboratory.

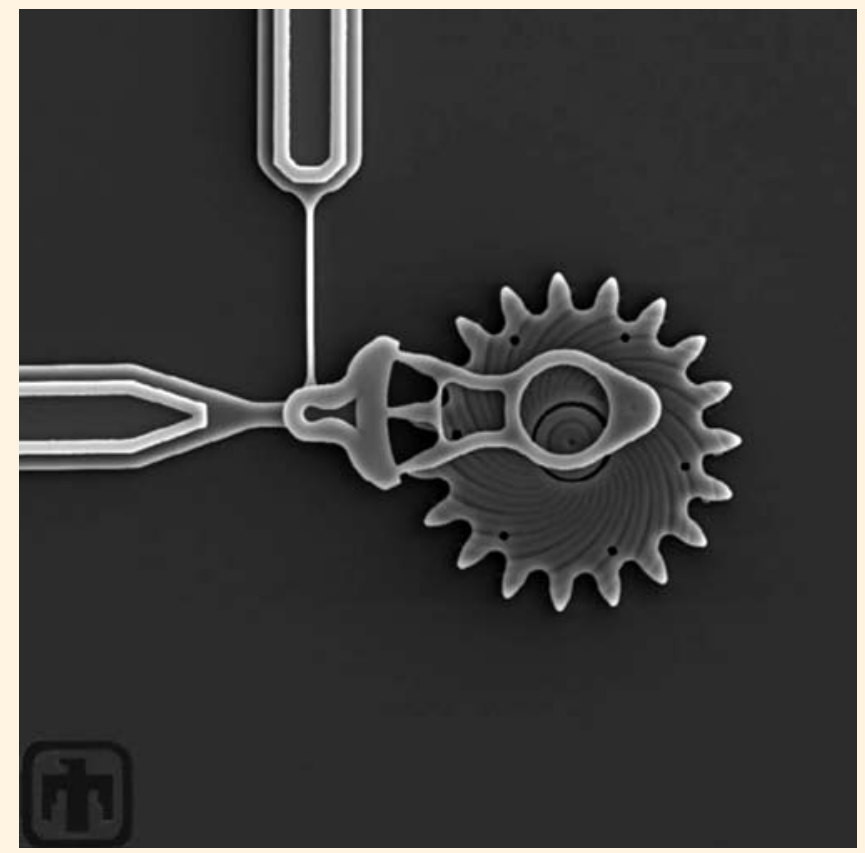

A las escalas que operan los mems, las fuerzas de Casimir juegan un papel importante. Recordemos de la sección de experimentos que el dispositivo en la figura 4 es de hecho una micromáquina. Con el dispositivo de dicha figura, la fuerza de Casimir sólo se puede medir hasta una distancia mínima de unos $5 \mathrm{~nm}$. ¿Por qué? Si en un dispositivo como el mostrado en dicha figura, acercáramos la esfera al plano por debajo de los $5 \mathrm{~nm}$ ocurre el llamado salto a contacto. La esfera y el plano se pegan y el dispositivo queda dañado permanentemente. En este caso hay dos fuerzas que actúan sobre la microbalanza de la figura 4. Una es la fuerza de Casimir y la otra es una fuerza de restitución elástica que trata de regresar la micro balanza a su posición horizontal que es la de equilibrio. Cuando la fuerza de Casimir es mayor que la elástica sucede el salto a contacto. Para ilustrar más claramente el efecto del salto a contacto en la figu- 
ra 8 mostramos un esquema de un sistema con las dos fuerzas compitiendo. La fuerza elástica, cuya energía potencial es la línea punteada y la fuerza de Casimir representada por la línea rayada. La suma de las dos es la línea sólida que muestra una barrera de potencial aproximadamente a $30 \mathrm{~nm}$ en la figura. Para todos los puntos a la derecha de esta barrera, la fuerza de Casimir es mayor que la fuerza elástica (precaución, la figura muestra energías potenciales no fuerzas) y se da el salto a contacto.

FIGURA 8. Energías potenciales elásticas y de Casimir. Todos los puntos a la derecha de la barrera de potencial en aprox. $30 \mathrm{~nm}$ son inestables.

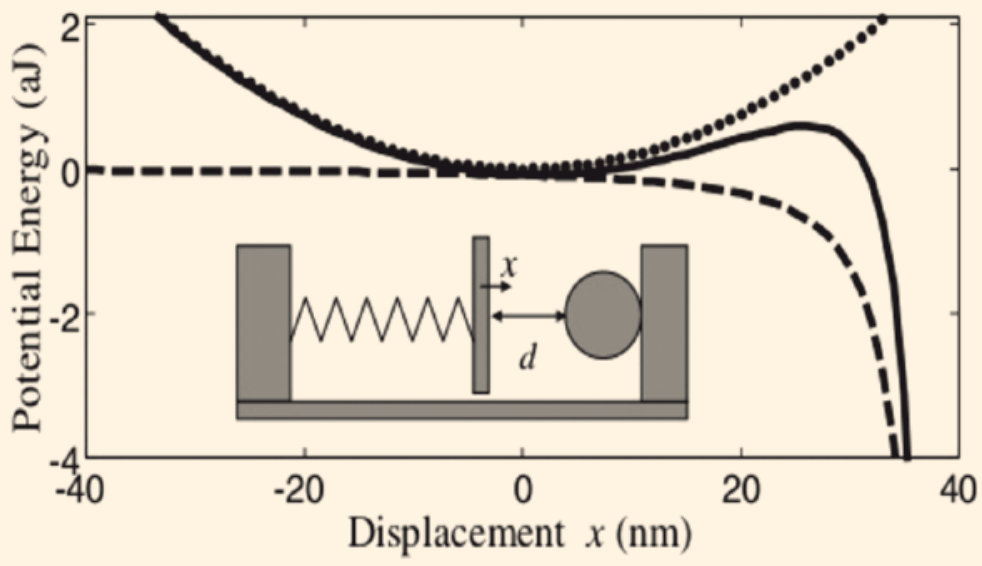

Para muchos dispositivos el salto a contacto es un efecto indeseable. Si bien la fuerza elástica se puede modificar cambiando las propiedades de los materiales, la fuerza de Casimir siempre está presente. Una pregunta es si es posible modificar o modular la fuerza de Casimir.

Una manera obvia de cambiar la fuerza de Casimir es cambiar el tipo de material, como mencionamos, es mayor si usamos metales. Esto resulta poco práctico por lo que se deben buscar otros métodos.

Recientemente se propuso que usar campos magnéticos externos podría utilizarse para alterar la fuerza de Casimir. Existen materiales semiconductores que al aplicarles un campo magnético cambian sus propiedades ópticas. En particular se hacen menos reflejantes. Recordemos que entre más reflejan mayor es la fuerza de Casimir, lo cual nos permite controlar la fuerza. El cambio en las propiedades ópticas de estos materiales se debe a unas excitaciones llamadas magneto plasmones de superficie. Por el momento, no entraremos en los detalles de estas excitaciones. Nos basta que cambien las propiedades ópticas y en particular que nuestro material refleje menos luz.

Esta idea de controlar la fuerza de Casimir con campos magnéticos externos fue propuesta en una colaboración entre la UNAM y la Universidad Autónoma de Puebla [10]. Usando semiconductores como InSb o AsGa se logró disminuir la fuerza de Casimir al aplicar un campo magnético paralelo a las superficies. En la figura 9 se puede ver cómo disminuye la fuerza de Casimir con el campo magnético. Para entender 
FIGURA 9. Factor de reducción definido en la ecuación 2 para distintos valores de $\Omega$ c. Al aumentar el campo magnético aumenta Ùc y disminuye la fuerza de Casimir. Figura tomada de la referencia [10].

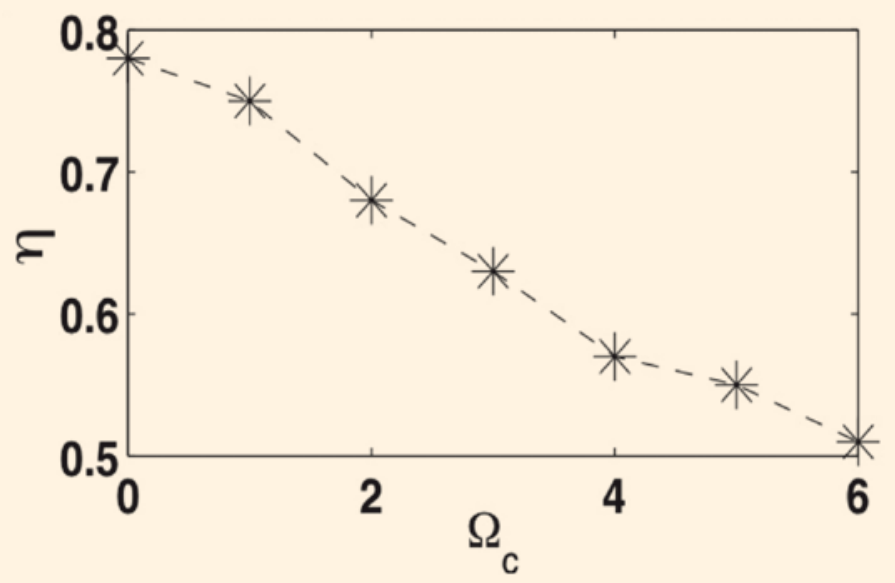

la figura hay que definir algunas cantidades: en el eje vertical graficamos el factor de reducción $\eta$ definido en la ecuación 2 , y en el eje horizontal graficamos $\Omega$ c que es una frecuencia adimensional directamente proporcional al campo magnético aplicado. Es decir, cuando el campo magnético es cero la frecuencia $\Omega$ c es cero y al aumentar el campo magnético, la frecuencia $\Omega$ c también aumenta.

Otra consecuencia inesperada de utilizar campos magnéticos para controlar la fuerza de Casimir, es la aparición de una torca de Casimir [11]. Como mencionamos, el aplicar un campo magnético externo cambia las propiedades ópticas de los materiales utilizados. En particular los hace ópticamente anisotrópicos. La anisotropía óptica es la causante de la torca del vacío. En la figura 10 mostramos un esquema donde

FIGURA 10. Dos placas paralelas ópticamente anisotrópicas . La torca de Casimir tiende a alinear los ejes de las placas. Tomada de la referencia [11].

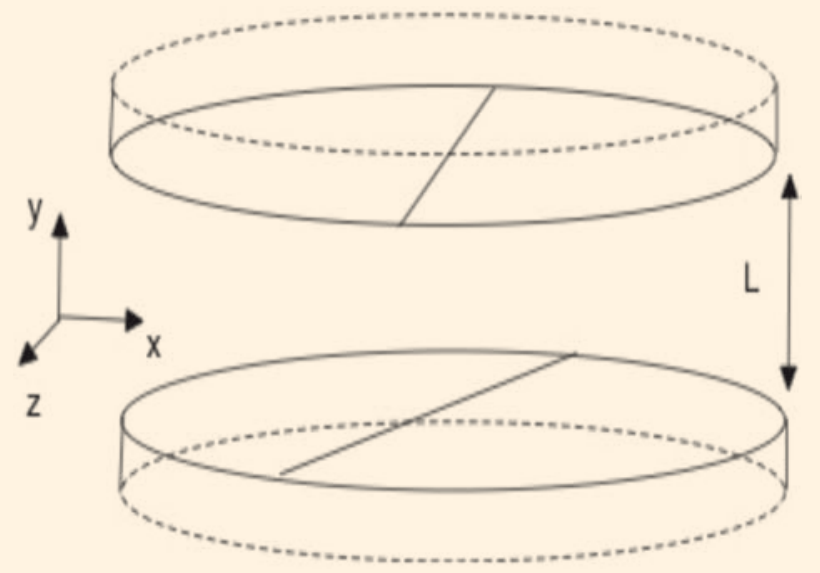


se ven dos placas paralelas anisotrópicas. La línea que se muestra en las placas indica una dirección preferencial como referencia para mostrar que sus ejes ópticos están rotados. La torca de Casimir tiene el efecto de alinear las dos placas.

La torca de Casimir se calcula considerando el cambio de energía para rotar una placa un cierto ángulo. Al igual que la fórmula de Lifshitz, el cálculo de la torca depende de las propiedades ópticas de las placas y del medio entre éstas.

De nuevo, al aplicar un campo magnético externo e inducir una anisotropía óptica se genera una torca de Casimir. En la figura 11 graficamos la torca entre dos placas para distintos valores del parámetro $\Omega$ c que, recordemos, es proporcional al campo magnético. Entre mayor es el campo magnético el valor de $\Omega$ c también se incrementa. Vemos que el valor de la torca va aumentando. Si bien parece que su valor es pequeño, con la tecnología de mems y nems es posible medirla.

FIGURA 11. La torca de Casimir como función del ángulo que rotan las placas para distintos valores del campo magnético.

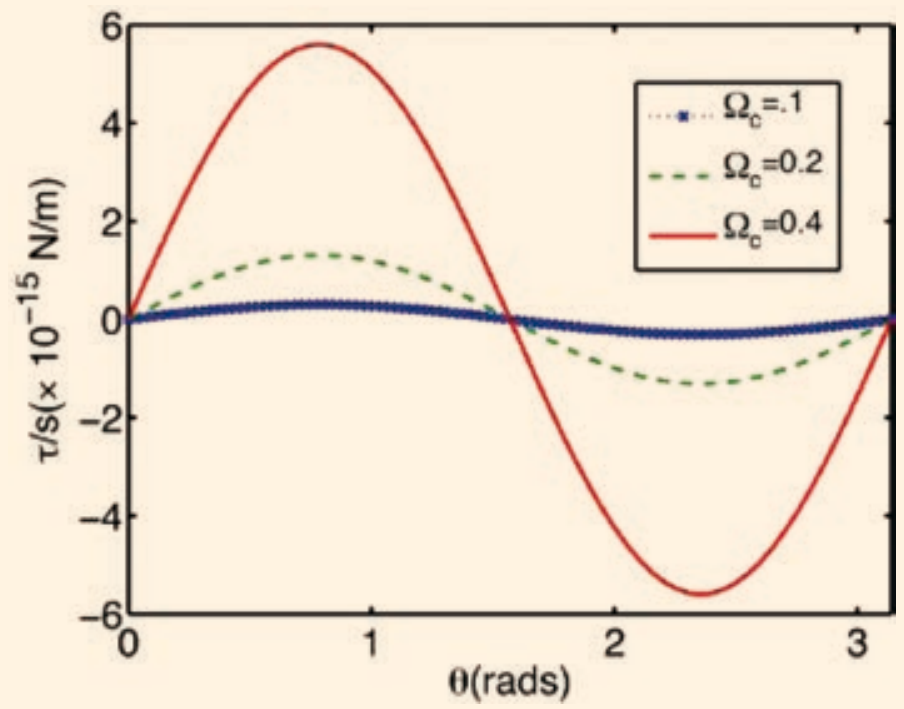

\section{Conclusiones}

Si bien la fuerza de Casimir parece un concepto oscuro de la física teórica, ha encontrado aplicaciones en diversas áreas como micro y nano máquinas, coloides, autoensamblaje, cosmología, altas energías por mencionar algunos. Gracias a los experimentos recientes, la comunidad científica ha retomado su interés en este tema quedando mucho por hacer. En particular, el poder tener mecanismos novedosos para controlar la fuerza de Casimir.

En este artículo se presenta un panorama reducido de la fuerza de Casimir. Un tratamiento muy detallado de esta fuerza se puede encontrar en la referencia [12]. 
Hay que tener cuidado con el concepto de energía del vacío. Desafortunadamente, con el gran acceso a la información del internet, varios sitios han difundido ideas erróneas sobre el vacío. En particular se habla de poder utilizar la energía del vacío como una fuete inagotable de energía. Esto no es posible gracias a las leyes de la termodinámica.

\section{REFERENCIAS}

[1] H. B. G. Casimir. (1948). "On the attraction between two perfectly conducting plates". Proc. Kon. Nederland. Akad. Wetensch. B51: 793.

[2] P. W. Milonni. (1994). "The Quantum Vacuum, an introduction to quantum electrodynamics. Academic Press Inc.

[3] J. P. Dowling. (1989). "The mathematics of the Casimir effect". Mathematics Magazine, 62, 324 .

[4] I. E. Dzyaloshinskii, E. M. Lifshitz y L. P. Pitaevskii. (1961). “General Theory of Van der Waals forces". Sov. Phys. Uspekhi, 73, 153.

[5] S. K. Lamoreaux. (1997). "Demonstration of the Casimir force in the 0.6 to 6 micron range". Phys. Rev. Lett. 78, 5.

[6] U. Mohideen y A. Roy. (1998). "Precission measurements of the Casimir force from 0.1 to 0.9 Microns". Phys. Rev, Lett. 81, 4549.

[7] H. B. Chan, V. A. Aksyuk, R. N. Kleiman, D. J. Bishop y F. Capasso. (2001). “Nonlinear micromechanical Casimir oscillator". Phys. Rev. Lett. 87, 211801.

[8] P. J. van Zwol, G. Palasantzas, M. van de Schootbrugge y J. Th. M. de Hosson. "Measurements of dispersive forces between evaporated metal surfaces in the range below $100 \mathrm{~nm}$ ". arXiv: 0712.3118.

[9] V. A. Parsegian. (2006). Van der Waals forces: A handbook for biologists, chemists, engineers, and physicists. Cambridge Univ. Press.

[10] R. Esquivel-Sirvent, M. A. Palomino-Ovando y G. H. Cocoletzi. (2009). Appl. Phys. Lett. 95, 051909.

[11] R. Esquivel-Sirvent, G. H. Cocoletzi y M. A. Palomino-Ovando. (2010). J. Appl. Phys. $108,114101$.

[12] M. Bordag, G. L. Klimchistkaya, U. Mohideen y V. M. Mostepanenko. (2009). Advances in the Casimir Effect. Oxford Science Publications. 